\title{
Determination of the deposition order of overlapping latent fingerprints and inks using Secondary Ion Mass Spectrometry (SIMS).
}

\begin{tabular}{|r|l|}
\hline Journal: & Analytical Chemistry \\
\hline Manuscript ID: & ac-2012-00185j.R1 \\
\hline Manuscript Type: & Article \\
\hline Author: & n/a \\
\hline Complete List of Authors: & $\begin{array}{l}\text { Bright, Nicholas; University of Surrey, Surrey Ion Beam Centre } \\
\text { Webb, Roger; University of Surrey, Ion Beam Centre } \\
\text { Hinder, Steven; University of Surrey, , The Surface Analysis } \\
\text { Laboratory, School of Engineering, } \\
\text { Kirkby, Karen; Surrey Ion Beam Centre, University of Surrey, } \\
\text { Ward, Neil I.; University of Surry, UK, Chemistry } \\
\text { Watts, John; University of Surrey, Faculty of Engineering \& Physical } \\
\text { Sciences } \\
\text { Bleay, Stephen; Home Office Scientific Development Branch, } \\
\text { Fingerprint and Footware Forensic Group } \\
\text { Bailey, Melanie; University of Surrey, Chemical Sciences }\end{array}$ \\
\hline
\end{tabular}

\section{SCHOLARONE ${ }^{\text {Th }}$ Manuscripts}




\title{
Determination of the deposition order of overlapping
}

\section{latent fingerprints and inks using Secondary Ion Mass}

\section{Spectrometry (SIMS).}

\author{
Nicholas. J. Bright ${ }^{1}$, Roger. P. Webb ${ }^{1}$, Stephen. Bleay ${ }^{2}$, Steven. Hinder ${ }^{3}$, Neil. I. Ward ${ }^{4}$, John. F. Watts ${ }^{3}$, \\ Karen. J. Kirkby ${ }^{1}$, and Melanie J. Bailey ${ }^{4 *}$
}

Surrey Ion Beam Centre, Advanced Technology Institute, Univ. of Surrey, Guildford, Surrey, GU2 7XH

N.Bright@Surrey.ac.uk

Corresponding Author:

*Melanie J. Bailey

Tel : 01483682593

Fax : 01483686091

Email : $\underline{\text { m.bailey@surrey.ac.uk }}$

Current addresses of other authors:

${ }^{1}$ Surrey Ion Beam Centre, University of Surrey, Guildford, Surrey, GU2 7XH

${ }^{2}$ Home Office Centre for Applied Science and Technology, St Albans, Hertfordshire, AL4 9HQ

${ }^{3}$ The Surface Analysis Laboratory, University of Surrey, Guildford, Surrey, GU2 7XH

${ }^{4}$ Chemical Sciences, University of Surrey, Guildford, Surrey, GU2 7XH 
Abstract

A new protocol using time-of-flight secondary ion mass spectrometry (ToF-SIMS) has been developed to identify the deposition order of a fingerprint overlapping an ink line on paper. By taking line scans of fragment ions characteristic of the ink molecules $(\mathrm{m} / \mathrm{z} 358.2$ and 372.2) where the fingerprint and ink overlap and by calculating the normalised standard deviation of the intensity variation across the line scan, it is possible to determine whether or not a fingerprint is above ink on a paper substrate. The protocol adopted works for a selection of fingerprints from four donors tested here and for a fingerprint that was aged for six months; for one donor, the very faint fingerprints could not be visualized using either standard procedures (ninhydrin development) or SIMS and therefore the protocol correctly gives an inconclusive result.

\section{Introduction}

Detecting and identifying a fingerprint on a handled document is a relatively straightforward task and in general requires only simple chemical reagents. However, when a fingerprint overlaps with an ink line (e.g. from a handwritten note) there is no reliable method to determine if the fingerprint has been laid prior to the ink being deposited, or whether the ink was laid down before the fingerprint (see Figure 1). Resolving this question is significant in criminal investigations where police may find a fingerprint on a document, but the suspect claims to have handled the paper before any ink was put down on the paper e.g. "I handled the paper before the document was written". This will also have implications when investigating historical artifacts, where the authenticity and relationship to an author/artist is questioned. 


\section{Page 3 of 18}

1

2

3

4

5

6

7

8

9

10

11

12

13

14

15

16

17

18

19

20

21

22

23

24

25

26

27

28

29

30

31

32

33

34

35

36

37

38

39

40

41

42

43

44

45

46

47

48

49

50

51

52

53

54

55

56

57

58

59

60
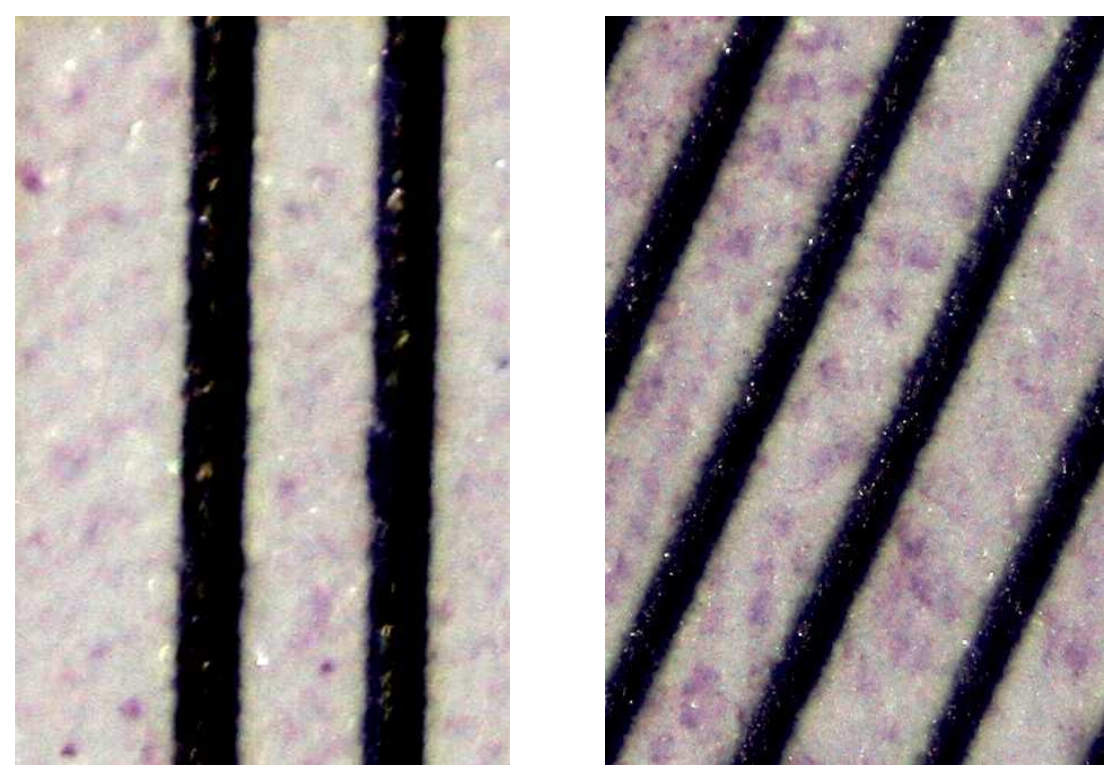

Figure 1. Optical images of a fingerprint deposited A) on top of ink and B) underneath ink, and then developed with ninhydrin. 
Latent fingerprints are a highly variable combination of chemicals that originate from (predominantly) natural secretions from pores found on the skin and from exogenous contaminants ${ }^{12},{ }_{3}, 4$. The natural components consist mainly of eccrine ${ }^{5}$ and sebaceous gland secretions ${ }^{6}$. Eccrine gland secretions from the fingers, hands, and soles of the feet contain both organic and inorganic species (amino acids and salts). The problem with the current fingerprint development reagents such as ninhydrin or 1,8Diazafluoren-9-one (DFO) is that they do not give any depth information (only a 2D image with light/dark contrast) ${ }^{78}$, This means that it is currently impossible to deduce whether a fingerprint was laid prior to or after ink was deposited on the document. The aim of this research is to investigate whether it is possible to identify if a fingerprint is below or on top of ink on a document using time-of-flight secondary ion mass spectrometry (ToF-SIMS). He et al. ${ }^{9}$ and Arnaud et al. ${ }^{10}$ have both used ToF-SIMS to establish the deposition order of overlapping writing inks on paper, but have not investigated overlapping fingerprints and inks. Previous work by Bailey et al. ${ }^{11}$ used a high energy variant of ToFSIMS (an experimental technique which is currently in the early stages of development and not widely available to the scientific community) to show that determining the deposition order of fingerprints and inks is potentially possible. However this study necessarily used fingerprints doped with hand cream to obtain a signal, which is not practical for most applications. Fieldhouse et al. ${ }^{12}$ reported that electrostatic detection apparatus (ESDA) could also potentially be used for the same purpose but this procedure could only be applied to LaserJet ink toners and not to pen inks. Using chemical information from molecules known to originate from fingerprints and inks to determine the deposition order is more selective than ESDA, which looks only at indentations in the surface of the sample. Hinder and Watts ${ }^{13}$ and Szynkowska et al. ${ }^{14}$ reported that ToF-SIMS can produce very high quality images of fingerprints on a range of materials (including carrier bags, printed newspaper, cling-film, metal and glass surfaces), whilst also providing information on the chemistry of a fingerprint. In this work, a novel protocol is presented for the determination of the depth ordering of fingerprints and inks on paper using ToF-SIMS.

\section{Experimental}


Fingerprint donors were asked to wash their hands thoroughly with soap and water (to keep exogenous species detected to a minimum). They were then told to carry out their normal activities (typically deskbound) for one hour in order to produce eccrine secretions on the fingertips. They were told not to eat but could consume water. To deposit fingerprints on the substrate the donor was told to use a medium touch pressure and to hold contact with the substrate for around half a second (i.e. a quick touch). Straight lines were drawn onto the paper (either before or after a fingerprint was deposited) using a Black Steadtler Stic 300M. The paper substrate was Discovery Nonstop Print Eco-Efficiency Paper (75 $\left.\mathrm{g} . \mathrm{m}^{-2}\right)$

ToF-SIMS analyses of latent fingerprints were carried out on an ION-TOF GmbH (Münster, Germany) TOF.SIMS.5 instrument. A $25 \mathrm{keV} \mathrm{Bi}_{3}{ }^{+}$primary ion beam focused to $\sim 4 \mu \mathrm{m}$ and delivering $0.18 \mathrm{pA}$ of current was employed with a primary ion dose density of $9.047 \mathrm{x} 10^{8}$ ions $/ \mathrm{cm}^{2}$ (this is substantially below the static limit). An electron flood gun $(20 \mathrm{eV})$ was used to prevent charge build up on the insulting sample surfaces. Images were acquired at $128 \times 128$ resolution in the MacroRaster mode of operation; in a MacroRaster scan the sample stage moves underneath the ion beam to produce composite images of areas covering $3.5 \mathrm{~mm} \times 3.5 \mathrm{~mm}$ with a pixel size of $27 \mu \mathrm{m}$ x $27 \mu \mathrm{m}$ (see the work of He et al ${ }^{9}$ and Pachuta et al. $^{15}$ for further demonstrations of MacroRaster scanning). The 128 resolution was selected as it provides good image resolution within an acceptable timeframe, i.e. just over 2 hours per image acquisition. Superior quality images can be acquired employing higher resolutions, but these require correspondingly longer acquisition times. Image data was acquired using 256 cycles per pixel point with 1 scan per pixel. A cycle time of $100 \mu$ s was employed. All ions referred to in this study were detected using a positive ToF-SIMS mode because previous ink analyses suggested that no additional information is provided from the negative spectrum ${ }^{16}$. The mass scale was calibrated using a method based on that described by Green et $a l^{17}$ and used the following positive ions $\mathrm{H}, \mathrm{CH}_{3}$, 
$\mathrm{CH}_{3} \mathrm{O}, \mathrm{C}_{3} \mathrm{H}_{3}, \mathrm{C}_{4} \mathrm{H}_{3}, \mathrm{C}_{5} \mathrm{H}_{11}, \mathrm{C}_{6} \mathrm{H}_{11}, \mathrm{C}_{7} \mathrm{H}_{13}, \mathrm{C}_{11} \mathrm{H}_{13}, \mathrm{C}_{24} \mathrm{H}_{28} \mathrm{~N}_{3}$ and $\mathrm{C}_{25} \mathrm{H}_{30} \mathrm{~N}_{3}$. The molecular ion images were normalised against the total ion image in order to account for any topography effects in the sample.

\section{Results and Discussion}

Figure 2 demonstrates that it is possible to determine whether a fingerprint has been laid prior to or after ink deposition by inspecting the ion images from specific molecules. Figure $2 \mathrm{~A}$ shows that when a fingerprint is deposited on top of an ink line, the ink molecules (m/z 358.2 and 372.2 - corresponding to dyes Basic Violet 1 and Basic Violet 3 respectively ${ }^{16}$ ) are masked in areas where the fingerprint ridges are found: determined from $\mathrm{m} / \mathrm{z} 88.1$ and 551.5 - believed to originate from butyric acid (a suspected fragment from various fatty acids found in fingerprints ${ }^{2}$ ) and a dimethyldioctadecylammonium ion (commonly found in personal care products ${ }^{18,19}$ ). Figure $2 \mathrm{~B}$ shows when an ink line is drawn across a fingerprint already deposited on paper, ions characteristic of the ink molecules (m/z 358.2 and 372.2) then mask and prevent the detection of those that are diagnostic of the fingerprint molecules (determined from $\mathrm{m} / \mathrm{z} 88.1$ and 551.5). The two mass fragments at 88.1 and 551.5 were chosen because both showed good image clarity of the fingerprint ridge detail. Many other masses were detected showing ridge detail but not all with the same clarity. 


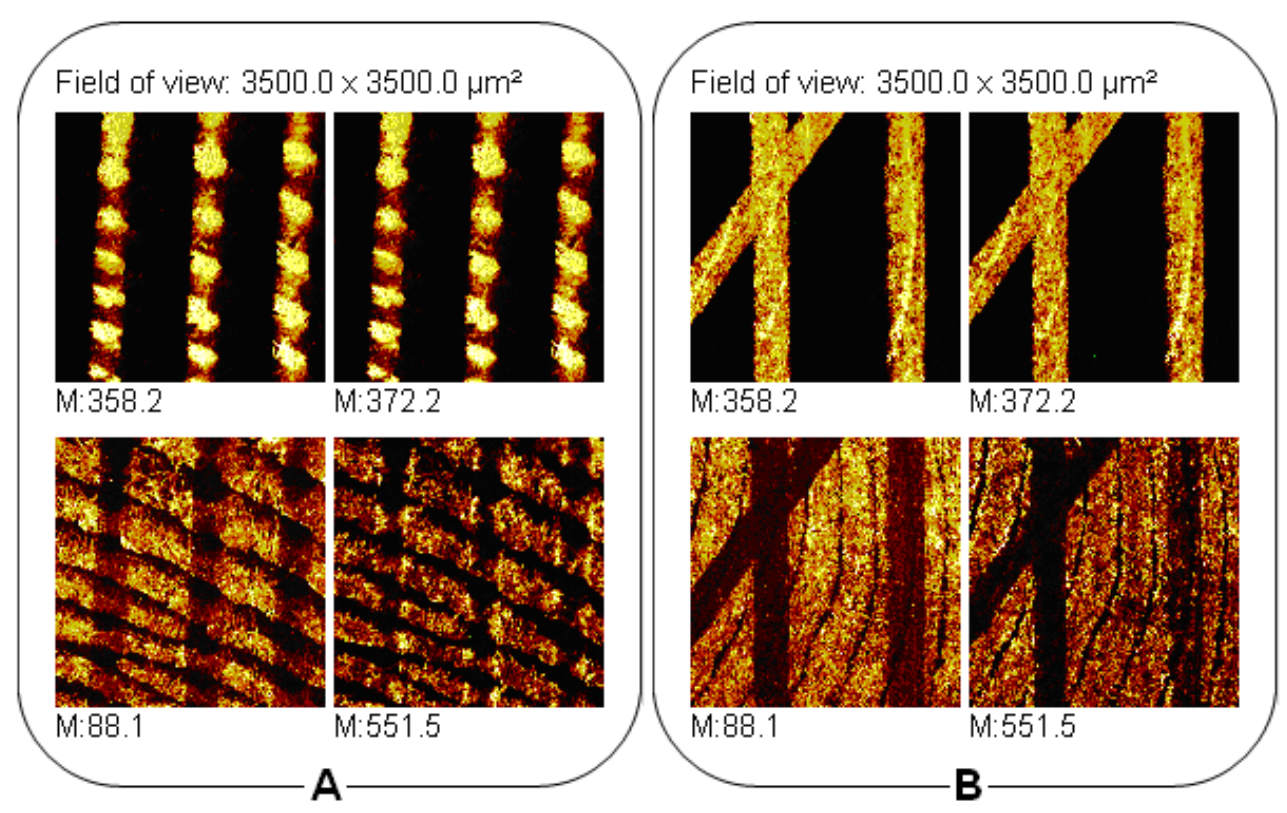

Figure 2. Positive ion images of a fingerprint deposited (A) after ink and (B) before ink on a paper substrate 
Figure 3 shows that the ion images of the ink molecules (when deposited before or after a fingerprint) can appear qualitatively similar to each other. Figure 3A shows that the fingerprint does not completely mask the ink and in fact it is still possible to see some ink ions from beneath the fingerprint. This is to be expected since fingerprint ridges can be seen to be made up of a series of droplets when viewed under an optical microscope and so will not uniformly cover the ink ${ }^{20}$. Conversely, Figure $3 \mathrm{~B}$ shows that when the ink line is deposited after the fingerprint, there are also variations in the intensity of the ink line. This is thought to be either due to a matrix effect of the fingerprint affecting the secondary ion yield of the ink molecules ${ }^{21,22}$, or due to the ink not adhering to the paper as well when the fingerprint is present. The net effect is that the ink line looks masked even when it is over the fingerprint. Therefore qualitative analysis of the images is subjective i.e. by utilizing the standard images alone it is not possible to determine the deposition order. A more rigorous approach must be developed in order to determine whether or not an ink line is above or below a fingerprint. 


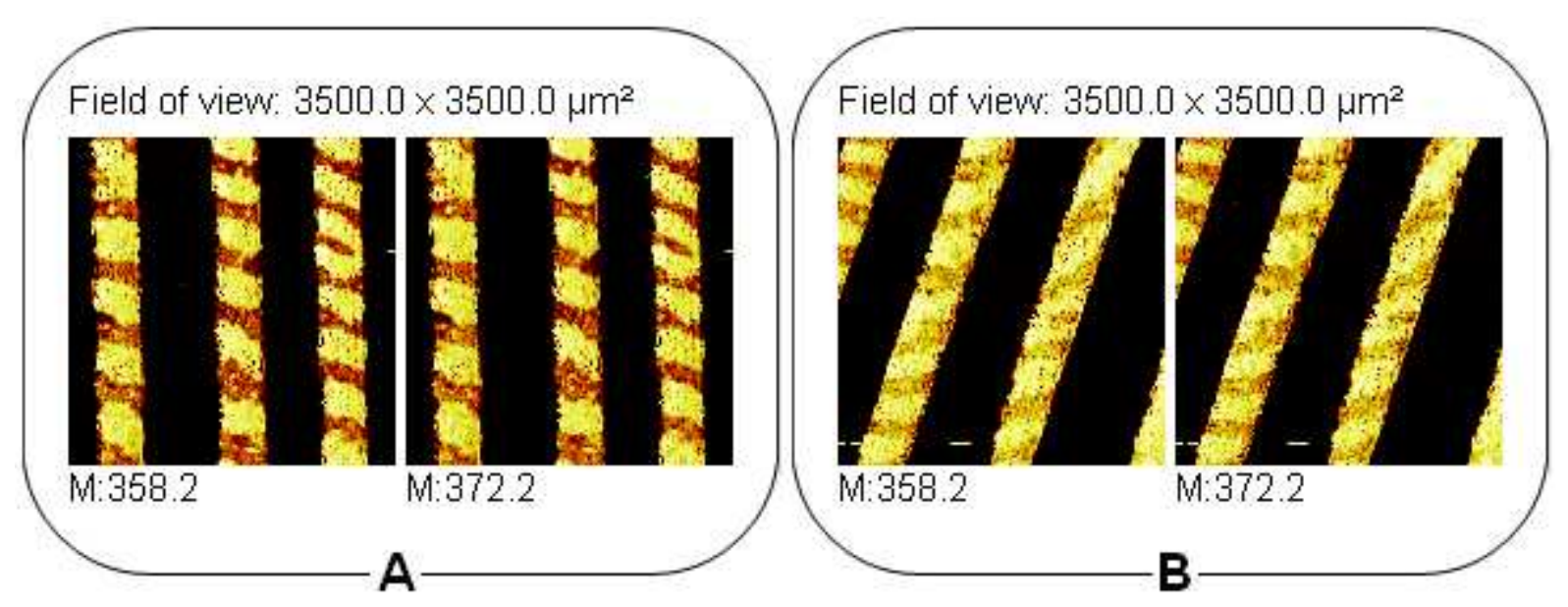

Figure 3. Positive ion images of a fingerprint deposited (A) after ink and (B) before ink on a paper substrate 
The protocol developed was to carry out line scans (post image analysis) along the ink lines in order to assess how the intensity of the molecules associated with the ink change on and off the fingerprint ridge. Line scan data was produced by analyzing the signal intensity of the peaks from $\mathrm{m} / \mathrm{z} 358.2$ and 372.2 at 128 points along a single ink line. The intensity of each point in the line scan was calculated using the weighted sum of the four surrounding pixels at the corresponding point in the image. Three line scans, approximately 4-5 pixels apart, were generated for each ink line and for each mass $(\mathrm{m} / z, 358.2$ and 372.2). Figure 4 shows the sum of the three line scans for one ink line using $m / z \quad 358.2$. The red line in Figure 4 corresponds to line scan data from a sample where the fingerprint (FP) lies on top of the ink. This is of a sinusoidal nature and is clearly more variable in intensity than the blue line (corresponding to a sample where the ink lies on top of the fingerprint (FP)). It therefore follows that the standard deviation of the line scan data could be used as an indicator of the sequence of fingerprint and ink. Due to unavoidable differences in operating conditions, such as primary ion current fluctuations, the collected signal for a given $\mathrm{m} / \mathrm{z}$ can be expected to vary from run to run. Therefore the amplitude of the variation in the line scan data would also be expected to vary from run to run. To account for this variation, the standard deviation is normalized to the root mean square (RMS) of the line scan data. 


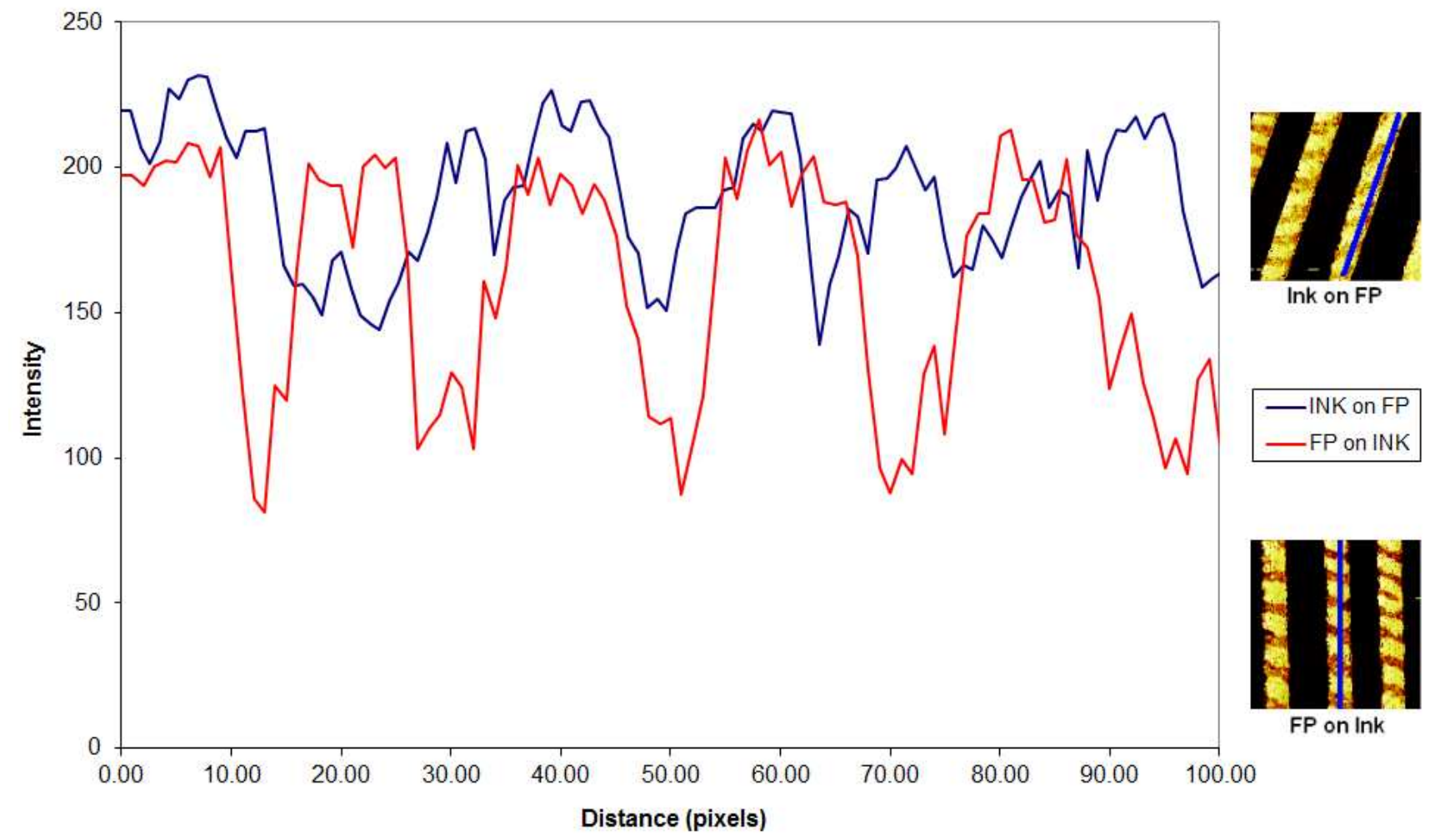

Figure 4. Line scans of overlapping fingerprints and inks following the intensity of ions characteristic of the ink (from $\mathrm{m} / \mathrm{z} 358.2$ ) - the inserted images show the location where the line scans were taken 
Figure 5 shows the normalized standard deviations of line scan data from two ink ions from 6 different fingerprints coming from 4 different donors (donor 1 depositing 3 different sets of fingerprints on different days and one of those sets being reanalyzed 6 months later), both above and below ink lines. Some samples only had one ink line available within the scan area, whereas others had multiple; where possible 3 ink lines per scan were analyzed. For all donors, the normalized standard deviation of the ink line scan is highest when the fingerprint is on top of the ink. However, when dealing with real case samples, an analyst will not necessarily be able to compare the questioned document to controlled samples deposited by the same donor. Therefore the following protocol is suggested, based upon the results generated in this study: if the normalized standard deviation is higher than 23.5 , the fingerprint is above the ink line; if the normalized standard deviation is less than 23.5 then the results are inconclusive. This is because a normalized standard deviation of less than 23.5 can arise for one of 2 reasons a) the ink line is unmasked and nothing lies on top of it or b) a fingerprint lies on top but does not mask the ink line sufficiently, presumably because insufficient material has been deposited in the fingerprint. For example, in the case of Donor 3, the protocol determined all the line scans to be inconclusive, but when this fingerprint was subsequently developed with ninhydrin, no ridge detail could be seen. A confidence limit of $98 \%$ of the data is obtained using the cut-off value of 23.5. This is derived by taking the mean plus $2 \sigma$ of the ink over fingerprint data (using both ink masses i.e. 358.2 and 372.2).

A limitation of the protocol is that it is only possible to conclude that a fingerprint is above text on a document, or that the result is inconclusive. The protocol does not allow the user to determine that a fingerprint is below text. However, locating a fingerprint above text carries substantially greater evidential value (showing the suspect touched a document after it was written) than below. 
1

2

3

4

5

7

8

9

10

11

12

13

14

15

16

17

18

19

20

21

22

23

24

25

26

27

28

29

30

31

32

33

34

35

36

37

38

39

40

41

42

43

44

45

46

47

48

49

50

51

52

53

54

55

56

57

58

59

60

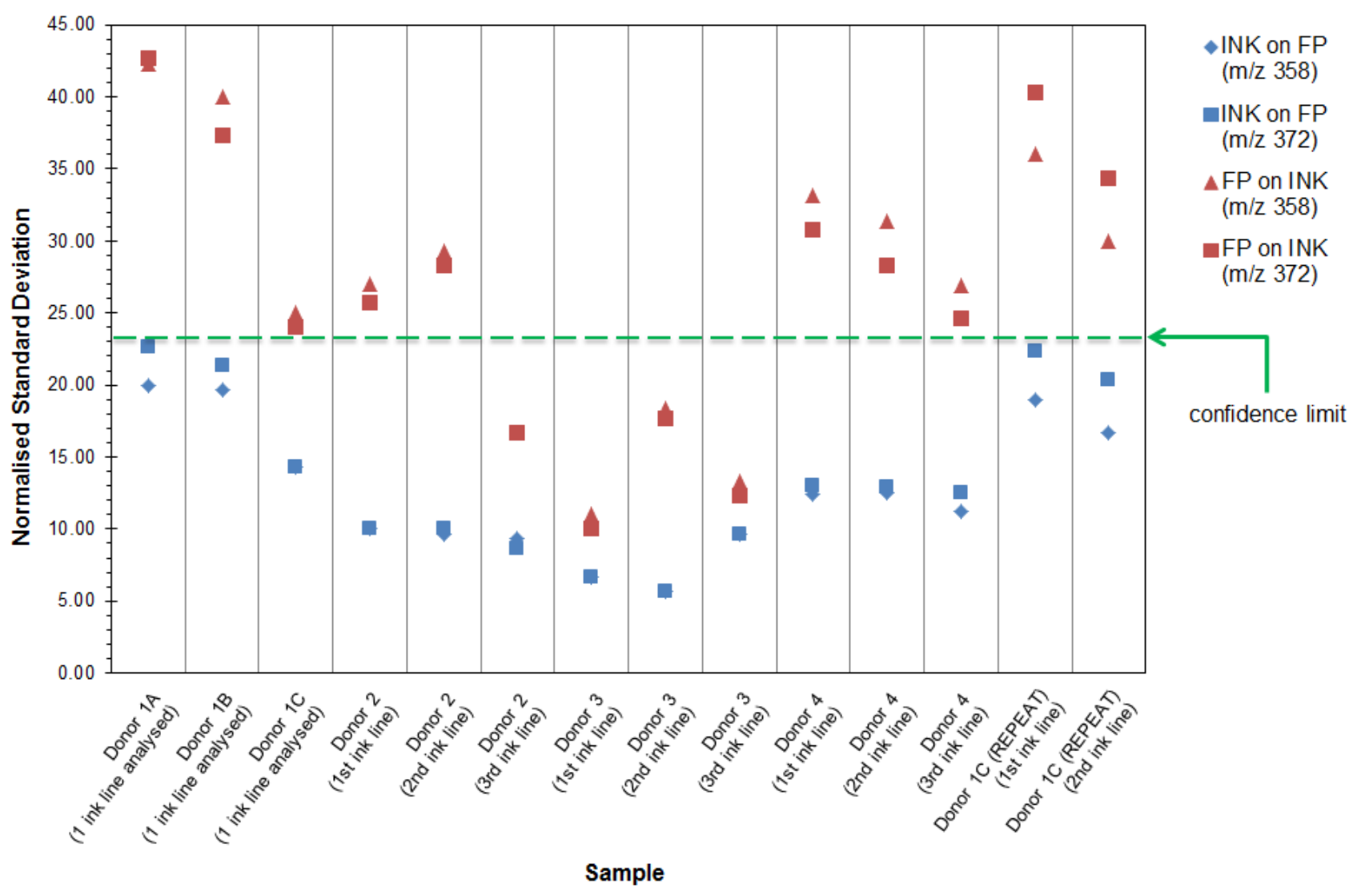

Figure 5: Normalized standard deviations of the line scan data for each of the different samples in the study, compared with the protocol "cut-off" point 
There are many variables which are known to affect the chemistry of a latent fingerprint including the donor, the age of the fingerprint and the environment to which the fingerprint has been exposed since deposition $^{2,23,24}$. This, and the uncontrolled nature of fingerprint deposition in police case work means that each time a fingerprint is deposited, differing substances in differing quantities will appear in the fingerprint residue. However, as mentioned previously, SIMS is sensitive to a range of different molecules (both endogenous and exogenous) in fingerprint residue and therefore imaging of the fingerprint using any one of these different compounds is normally possible. However, due to these uncertainties, an important aspect of the protocol is that it uses the ink rather than fingerprint images to determine the deposition sequence. The peaks at m/z 358.2 and 372.2 - corresponding to dyes Basic Violet 1 and Basic Violet 3 - are found in many blue / black pen inks ${ }^{16}$ and therefore are expected to be present in a large number of cases. Further work is ongoing to determine the protocol for inks not containing these dyes

In order to carry out the SIMS analysis, the sample is necessarily placed under ultra-high vacuum (10 ${ }^{10}$ Torr) and it is anticipated that this would artificially enhance the ageing process of the fingerprint, with the evaporation of the volatile components and migration of mobile species. In Figure 5 it can be seen that by reanalyzing one of the samples (6 months after the original analysis) the same result is achieved. This would suggest that any volatile or mobile species that are removed in the vacuum system or by ageing do not contribute to the masking of the ink ions.

\section{Conclusions}

A new protocol using time-of-flight secondary ion mass spectrometry (ToF-SIMS) has been developed to identify the deposition order of a fingerprint and a ballpoint pen ink line. By carrying out line scans of the ink molecules and by calculating the normalized standard deviation of the intensity variation along the line scan, it is possible to determine whether a fingerprint is above ink on a paper 
substrate (straightforward ToF-SIMS imaging alone is insufficient). The work presented here used only undeveloped latent fingerprints, however, it is normal for fingerprints to be developed first by investigators; the development techniques used by forensic investigators might well affect the analysis presented here and this will be the subject of a future paper.

\section{Acknowledgements}

The authors thank EPSRC for funding Nicholas Bright's Doctoral Training Account.

References

1. Buchanan, M. V.; Asano, K.; Bohanon, A., J. Soc. Photo-Opt. Instrum. Eng. 1997, 2941, 89 - 95.

2. Croxton, R. S.; Baron, M. G.; Butler, D.; Kent, T.; Sears, V. G., Forensic Sci. Int. 2010, 199, 93 $-102$.

3. Mong, G. M.; Petersen, C. E.; Clauss, T. R. W. Advanced Fingerprint Analysis Project Fingerprint Constituents; PNNL-13019; R\&D Project: 28471; Pacific Northwest National Laboratory Report: 1999; p 48

4. Asano, K. G.; Bayne, C. K.; Horsman, K. M.; Buchanan, M. V., J. Forensic Sci. 2002, 47, 805 807.

5. Worley, C. G.; Wiltshire, S. S.; Miller, T. C.; Havrilla, G. J.; Majidi, V., J. Forensic Sci. 2006, $51,57-63$.

6. Antoine, K. M.; Mortazavi, S.; Miller, A. D.; Miller, L. M., J. Forensic Sci. 2010, 55 (2), 513 518.

7. Lennard, C., The Detection and Enhancement of Latent Fingerprints. In 13th INTERPOL Forensic Science Symposium, Lyon, France, October 16-19, 2001. 
8. Bowman, V., Manual of Fingerprint Development Techniques. 2nd ed.; Home.Office.Scientific.Development.Branch, Ed. White Crescent Press Ltd: Luton, 2004.

9. He, A.; Kapusov, D.; Xu, S., Surf. Interface Anal. 2006, 38, 854 - 858.

10. Arnaud, C. H., Chemical \& Engineering News 2011, 89 (23), 42.

11. Bailey, M. J.; Jones, B. N.; Hinder, S.; Watts, J. F.; Bleay, S.; Webb, R. P., Nucl. Instrum. Methods Phys. Res., Sect. B 2010, 268, 1929 - 1932.

12. Fieldhouse, S. J.; Kalantzis, N.; Platt, A. W. G., Forensic Sci. Int. 2011, 206 (1-3), 155 - 160.

13. Hinder, S. J.; Watts, J. F., Surf. Interface Anal. 2010, 42 (6), 826 - 829.

14. Szynkowska, M. I.; Czerski, K.; Rogowski, J.; Parykczak, T.; Parczewski, A., Forensic Sci. Int. $2009,184,24-26$.

15. Pachuta, S. J.; Vlasak, P. R., Analytical Chemistry 2012, 84 (3), 1744-1753.

16. Coumbaros, J.; Kirkbride, K. P.; Klass, G.; Skinner, W., Forensic Sci. Int. 2009, 193, 42 - 46.

17. Green, F. M.; Gilmore, I. S.; Seah, M. P., J. Am. Soc. Mass Spectrom. 2006, 17 (4), 514 - 523.

18. Wolstenholme, R.; Bradshaw, R.; Clench, M. R.; Francese, S., Rapid communications in mass spectrometry 2009, 23, $3031-3039$.

19. Manier, M.; Cornett, D.; Hachey, D.; Caprioli, R., J. Am. Soc. Mass Spectrom. 2008, 19 (5), 666 -670 .

20. Thomas, G. L., J. Phys. E: Sci. Instrum. 1978, 11 (8), 722 - 731.

21. Leggett, G. J.; Vickerman, J. C., International Journal of Mass Spectrometry and Ion Processes 1992, $122(0), 281-319$. 
22. Leggett, G. J.; Vickerman, J. C.; Briggs, D.; Hearn, M. J., Journal of the Chemical Society, Faraday Transactions 1992, 88 (3).

23. Archer, N. A.; Charles, Y.; Elliot, J. A.; Jickells, S., Forensic Sci. Int. 2005, 154, 224 - 239.

24. Payne, G.; Reedya, B.; Lennard, C.; Comberb, B.; Exline, D. L.; Roux, C., Forensic Sci. Int. $2005,150,33-51$. 
For TOC only

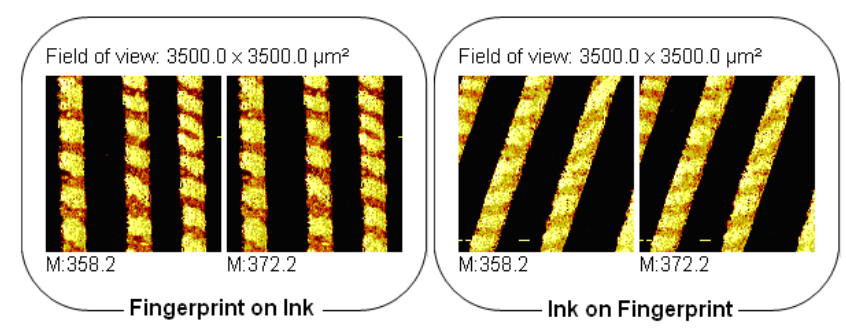

